\title{
Hall Mobility Field Effect in Two Layer Conductivity Samples
}

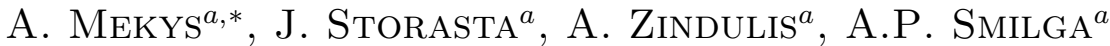 \\ AND S. BALAKAUSKAS ${ }^{b}$ \\ ${ }^{a}$ Institute of Materials Science and Applied Research \\ Vilnius University, Sauletekio 9-III, 10222 Vilnius, Lithuania \\ ${ }^{b}$ Institute of Semiconductors, A. Goštauto 11, LT-01108 Vilnius, Lithuania
}

(Received November 27, 2007; revised version March 28, 2008; in final form July 23, 2008)

\begin{abstract}
Classical Hall mobility experimental setup was applied for samples with parallel plane (sandwich) variable conductivity layers. The measured effective Hall mobility strongly depends on applied electric field and does not characterise the real carrier mobility. Numerical modelling explains the effect as a consequence of electric field redistribution and lowering at Hall contacts. Measurement of carrier mobility in such structures is suggested.
\end{abstract}

PACS numbers: $84.37 .+\mathrm{q}$

\section{Introduction}

The idea for this investigation came from the fact that the Hall mobility changed in wide range after soft X-ray irradiation with contradictory results [1]. The X-ray irradiation was originally used for point defect creation and stimulated diffusion in silicon crystals. The change of bulk parameters was most important, but as parasitic effect, sample showed the electric contact degradation if the radiation was applied on planar contacts. To avoid this, the radiation was applied on the opposite sample surface. Irradiation creates defects that act like dopants and ionised scattering centres. In some cases [1] soft X-rays could not penetrate through the whole sample and the plane with electrodes remained unchanged during irradiation and only after a while, diffusing defects filled the whole sample. The changes in $I-V$ measurements could be explained by defect doping, however, the calculated Hall mobility decreased much more and also it showed strong sensitivity to applied voltage.

*corresponding author; e-mail: algirdas.mekys@ff.vu.lt 
Several models for the Hall mobility coefficients in non-homogeneous samples are presented in [2-5] (in crystals with insulating inclusions) or [6,7] (also conductive inclusions), or in [8] certain geometrical sample configurations, which reduce electric current shorting effects, but none of these models can suggest experimental behaviour if there is no possibility to compare different samples or to analyse completely the structure if parameter stratification in a crystal is unavoidable. Complete devices, like MOSFETs, were already tested with the Hall technique in [9], and the field effect on the Hall voltage was not observed, still in the nearest future it can appear. The problem of mobility measurement in multilayers was originally studied by Petritz in [10], who considered $n$-type inversion layer on a $p$-type substrate. The study of the two layer interacting configurations was continued in [11]. Recently, GaN thin layers were tested using the Hall effect [12] and the parameters were compared with optically obtained from near to the surface layers. The photo-measurements could extract information about the material from the surface regions where the light was absorbed and it was not important if the sample deeper inside, which has been formed with the buffer layer between sapphire substrate and GaN layer, had greater conductivity. However, electrical parameters extraction (like carrier mobility) can be complicated in such structures and certain conditions have to be fulfilled for reliable results.

In this work it will be shown that electric field variation is useful in the Hall measurements in the cases when the investigated material has a hidden conductive layer, which is usual in crystals of epitaxial growth, where the buffer layer can be more conductive than the bulk. Experimental and computer modelling study was applied on silicon samples with conductive aluminium layer. Also, the Hall mobility measurements for high resistivity samples were performed in various electric fields.

\section{Sample fabrication and experimental setup}

Silicon $n$-type $(\rho=360 \Omega \mathrm{cm})$ bar $\left(7 \times 2 \times 0.3 \mathrm{~mm}^{3}\right)$ sample with planar ohmic contacts on one side was used in our experiments. Aluminium (for conductive layer) was evaporated on the opposite side to cause electric potential redistribution. Metal evaporation $(\approx 0.25 \mu \mathrm{m})$ was performed in vacuum chamber, sample was heated till $400^{\circ} \mathrm{C}$ for several minutes to remove water monolayer, then cooled down to $80^{\circ} \mathrm{C}$ before metal coverage. The Hall and $I-V$ measurements were performed at room temperature $(298 \mathrm{~K})$ before and after $\mathrm{Al}$ evaporation. Constant $1.7 \mathrm{~T}$ magnetic induction field was used in the Hall and magnetoresistance measurements.

\section{Results and discussion}

In silicon sample with aluminium layer calculated from experimental data effective Hall mobility (EHM - explanation is given further in this paragraph, now let us consider it as the Hall mobility) changes from $750 \mathrm{~cm}^{2} /(\mathrm{V} \mathrm{s})$ at lower 


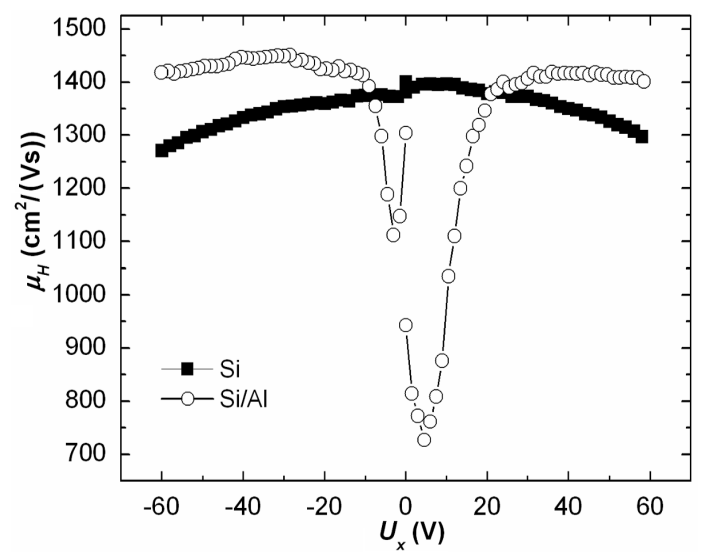

Fig. 1. Effective Hall mobility vs. applied voltage measurement. Open circles represent sample with Al layer, closed - without Al. Asymmetry of $\mu_{\mathrm{H}}$ near $U_{X}=0 \mathrm{~V}$ is a consequence of inhomogeneous sample coverage with $\mathrm{Al}$ in length: during metal evaporation sample holders shadowed one sample side more than the opposite one.

voltages and above $20 \mathrm{~V}$ it saturates to $1400 \mathrm{~cm}^{2} /(\mathrm{V} \mathrm{s}$ ) (which is even higher than for sample without Al layer as shown in Fig. 1). The Hall mobility for the sample with length $L$, width $w$ in magnetic induction $B$ field is calculated as

$$
\mu_{\mathrm{H}}=\frac{L}{w B} \frac{U_{\mathrm{H}}}{U_{X}} .
$$

In this formula, $U_{\mathrm{H}}$ is the Hall voltage, $U_{X}$ is taken as applied voltage on contacts and is not measured in the middle of the sample. That measurement requires precise technique, but in some sample configurations, like van der Pauw, it is too complicated. In our case (as the experiment shows in Fig. 1) it can be circumvent by measuring the range in constant magnetic field till $U_{\mathrm{H}} / U_{X}$ reaches saturation and does not further depend on $U_{X}$. In non-homogeneous samples electric field $U_{X} / L$ has to be taken locally, fortunately at the $U_{\mathrm{H}} / U_{X}$ saturation, $U_{X} / L$ locally becomes the same as for the whole sample. This is clear from the fact that the Hall voltage then becomes the same for homogeneous and inhomogeneous samples (Fig. 1). Homogeneous sample ensures that electric field locally is the same as for the whole sample. Also, for the sample with conductive layer it is not enough to measure local electric applied field $\left(U_{X} / L\right)$, because the Hall field $\left(U_{\mathrm{H}} / w\right)$ is directly shorted by the conductive layer and the Hall field has to be measured locally too, but that requires additional contacts, which are undesirable. Since neither electric field created by the power source nor by the Hall effect are accurately known, the Hall mobility is called effective (EHM) and from the experimental data EHM can be used for carrier mobility calculation only when EHM saturates (Fig. 1).

The modelling illustrating the electric field $\boldsymbol{E}$ redistribution in layered sample is based on carrier conservation and the Poisson equations in the stationary 
case $(\partial / \partial t=0)$ :

$$
\begin{aligned}
& \frac{\partial n}{\partial t}-\frac{\operatorname{div}\left(\boldsymbol{j}_{n}\right)}{q}=G_{n}-r n p-\gamma_{n} n, \\
& \frac{\partial p}{\partial t}+\frac{\operatorname{div}\left(\boldsymbol{j}_{p}\right)}{q}=G_{p}-r n p-\gamma_{p} n, \\
& \boldsymbol{j}_{n}=q n \boldsymbol{M}+q D_{n} \operatorname{grad}(n), \\
& \boldsymbol{j}_{p}=q p \boldsymbol{N}-q D_{p} \operatorname{grad}(p), \\
& \boldsymbol{M}=\frac{\mu_{n} \boldsymbol{E}-\mu_{n}^{2}[\boldsymbol{E}, \boldsymbol{B}]+\mu_{n}^{3} \boldsymbol{B}(\boldsymbol{B}, \boldsymbol{E})}{1+\mu_{n}^{2} B^{2}}, \\
& \boldsymbol{N}=\frac{\mu_{p} \boldsymbol{E}+\mu_{p}^{2}[\boldsymbol{E}, \boldsymbol{B}]+\mu_{p}^{3} \boldsymbol{B}(\boldsymbol{B}, \boldsymbol{E})}{1+\mu_{p}^{2} B^{2}}, \\
& -\operatorname{div}(\boldsymbol{E})=\Delta \varphi=-\frac{q(p-n+D)}{4 \pi \varepsilon \varepsilon_{0}} .
\end{aligned}
$$

Here $n, p-$ electron and hole densities, $\mu_{n}, \mu_{p}$ - mobilities, $\boldsymbol{j}_{n}, \boldsymbol{j}_{p}$ - current densities, $\gamma_{n}, \gamma_{p}$ - carrier trapping (mobile particles vanishing rate), $r$ - recombination, $G_{n}, G_{p}$ - generation, $D_{n}, D_{p}$ - diffusion coefficients, $q$ - absolute value of electron charge, $D$ - doping density (with charge sign). $\boldsymbol{M}$ and $\boldsymbol{N}$ calculated from the classical particle motion in magnetic field: $m \frac{\mathrm{d} \boldsymbol{v}}{\mathrm{d} t}=q \boldsymbol{E}+q[\boldsymbol{v}, \boldsymbol{B}]-\frac{\boldsymbol{v}}{\tau}$ with effective mass $m$, average velocity $\boldsymbol{v}$ and damping parameter $\tau$, the mobility expressed as $\mu=q \tau / m$. In computer modelling the sample is mounted into insulating media. This specifies boundary conditions: $n=p=\mu_{n}=\mu_{p}=0$ around the sample, i.e. carriers cannot leave the sample, because they are immobile outside. The ohmic contacts require that charge is not accumulating: $n p-n_{\mathrm{i}}^{2}=0$, $p-n+D=0\left(n_{\mathrm{i}}\right.$ - intrinsic) [13], the potential around the insulator is free to choose if its gradient is 0 (that boundary condition satisfies Eqs. (3.2)-(3.8) without introduction of new terms and physically it means that electric field vanishes far away from the sample). In this case constant value is taken: $1 / 2$ of the applied voltage, since then the calculating error is the smallest. The conductive layer for modelling is made with additional doping $(D)$. For simplicity shallow doping is assumed - generation and recombination through dopants is neglected and only intrinsic generation (equal to bipolar recombination at equilibrium) is left: $G_{n}=G_{p}=r n_{\mathrm{i}}^{2}$. The other parameters for modelling: distance between the nearest elements of space grid $-1 \times 10^{-7} \mathrm{~m}$ (it has to be shorter than the Debye screening length [14]), recombination coefficient $r=1 \times 10^{-10} \mathrm{~m}^{-3} \mathrm{~s}^{-1}$, maximal $n$-type doping $D=1 \times 10^{21} \mathrm{~m}^{-3}$, intrinsic concentration $n_{\mathrm{i}}=1 \times 10^{17} \mathrm{~m}^{-3}$, magnetic field induction $B=1 \mathrm{~T}$. For simplicity traps $\left(\gamma_{n}, \gamma_{p}\right)$ were eliminated, carrier mobilities were taken 1000 for electrons and $400 \mathrm{~cm}^{2} /(\mathrm{V} \mathrm{s})$ for holes [15]. 


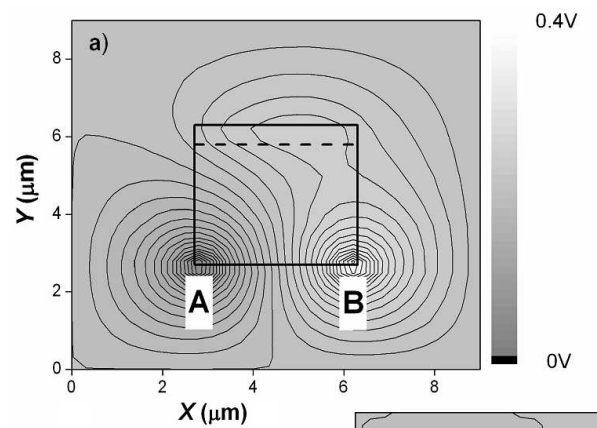

$0.4 \mathrm{~V}$

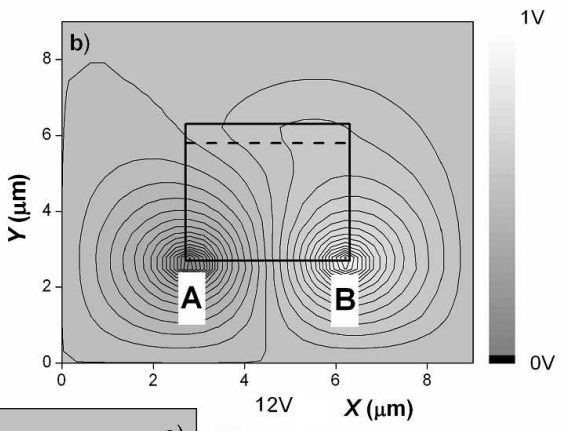

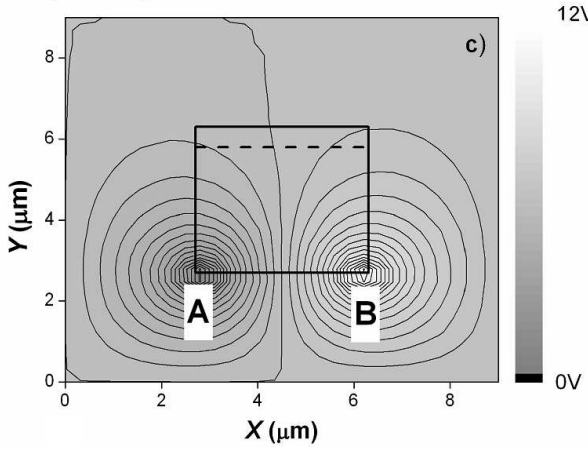

Fig. 2. The calculated equipotential lines in insulating sample with conductive layer at different applied voltages: (a) $0.4 \mathrm{~V}$, (b) $1 \mathrm{~V}$, (c) $12 \mathrm{~V}$. $X$ and $Y$ represent 2 spatial dimensions (one point equals to $1 \times 10^{-7} \mathrm{~m}$ ), inner rectangle is the sample (with electrodes connected at points $\mathrm{A}$ and $\mathrm{B}$ ), dashed line inside separates conductive $0.5 \mu \mathrm{m}$ thickness region (upper) from intrinsic (lower).

The modelled potential redistribution for several voltages is shown in Fig. 2a-c. The sample is marked as inner rectangle (surrounded by insulating media) with applied voltage at contact points A and B. Dashed line represents the separation of doped and intrinsic regions. In part (a) the applied voltage is low and the potential in the conductive layer has asymmetrical drop between the contacts because the conductive layer is separated by the junction barrier, which acts like diode, connected in reverse direction for the incoming current to the layer and in forward direction for outgoing current. When the applied voltage is low, the current through the barrier is near the same as through the rest of the sample. Between the points A and B the electric field is lower than it would be in the sample without the conducting layer. Figure $2 \mathrm{~b}$ is transitional to (c) where the conductive layer is not affecting any more because the major part of the current goes through the bulk of the sample and relatively small part through the barrier. The modelled effective Hall mobility is shown in Fig. 3 with different conductive layer doping. The modelling brings the same results as experiment. The values here are not important, since the modelling is only qualitative (Figs. 3, 4). The comparisons quantitatively should be done only for the same graph curves 


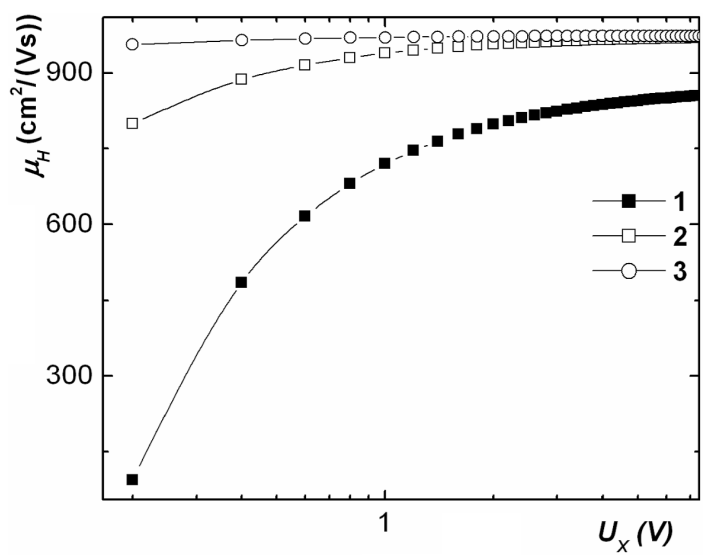

Fig. 3. Modelled effective Hall mobility (defined in (3.1) with $B=1 \mathrm{~T}$, and $L=w)$ dependence on applied voltage for different conductive layer $n$-type doping: $1-10^{20} \mathrm{~m}^{-3}, 2-10^{19} \mathrm{~m}^{-3}, 3-10^{18} \mathrm{~m}^{-3}$.

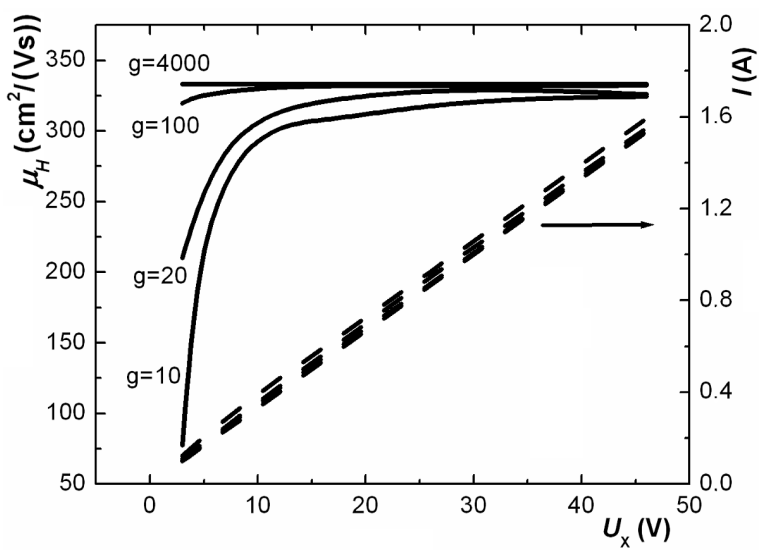

Fig. 4. Modelled Hall mobility (defined in (3.1) with $B=1 \mathrm{~T}$, and $L=w$ : left axis - solid lines) and $I-V$ (right axis - dashed lines) for the sample with conductive layer and different diode blocking parameters $g$ (shown numbers on the graph). The greater is $g$, the lower electric current can flow through the diodes. When $g=4000$, the conductive layer is completely (at current precision) isolated from the rest of the sample.

(they differ by conductive layer strength) and the comparison between the graphs is qualitative only. It has to be noted here that quantitative results of modelling cannot be obtained consistent with experimental at such low doping. In Eqs. (3.6), (3.7) for precise three-dimensional calculation all terms with $\mu^{2}$ and $\mu^{3}$ have to be included, and that requires enormously great matrixes and it becomes a waste of calculation time. Since $\mu^{2}$ and $\mu^{3}$ are the 2 nd and the $3 r d$ order small values, 
boundary values of electric potential strongly affect their contribution to the Hall voltage and the real mobility becomes difficult to find. Therefore only proportional values were found. For simplicity 2 -dimensional case was analysed with $90 \times 90$ point matrix and the Hall field was calculated from the local field and mobility as $E_{\mathrm{H}}=\mu E_{\text {local }} B$. In this model direct Hall field shorting by the conductive layer was not taken into account because it required one more dimension for calculation.

It is worth noting that in the stationary case the only information about the conductive layer is that it exists or not: electric field redistribution is determined by layer conductivity no matter it has greater or lower mobility. The Hall mobility voltage dependence does not give a scan of mobility through the sample depth.

Another modelling is based in Fig. 5. It is much easier for parameter picking and much faster for calculations, but not as informative as the previous. Here, diodes represent the Schottky barrier formed with aluminium evaporated on silicon from the other side than the contacts. At the points "A" and "B" voltage drop is not linearly proportional to applied voltage (on "+" and "-") because diodes introduce non-linearity. With weak voltages, diodes leak the current and voltage drop at $\mathrm{A}$ and $\mathrm{B}$ is small comparable to when two diodes block the current.

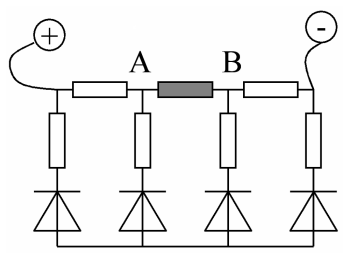

Fig. 5. Sample model with conductive layer and barrier on the back. Sample is connected at "+" and "-", the rectangles are the resistors, which represent sample parts where electric current flows. Diodes are important only when the leakage current is comparable to the current through the resistors. Diodes forward connection works as a Schottky barrier, the short connection between them stands for conductive layer.

In Fig. 4 Hall mobility is calculated vs. applied voltage for Fig. 5 taking $10 \Omega$ for each resistor and $30 \Omega$ for the darker one. The current through diode is given by the Shockley law: $I=\left[\exp \left(-q U_{\text {diode }} / k T\right)-1\right] / g$, here $g$ is the number given in the graph in Fig. 4 which stands for diode blocking parameter (it includes diode barrier height), $U_{\text {diode }}$ - voltage at diode. At greater voltage values the Hall voltage becomes linearly proportional to applied voltage and there the Hall mobility can be calculated precisely. $I-V$ does not show that great difference because the leak through diodes is comparable with the leak through resistors at low voltages.

The Hall effect sensitivity against $I-V$ in experiment (Fig. 6), explained as the Hall voltage, is an additive parameter to zero ("0") when the magnetic field is absent. In theory, the voltage at the Hall contacts is "0", and when the magnetic field is turned on, any Hall voltage value appearing at the contacts is much greater 


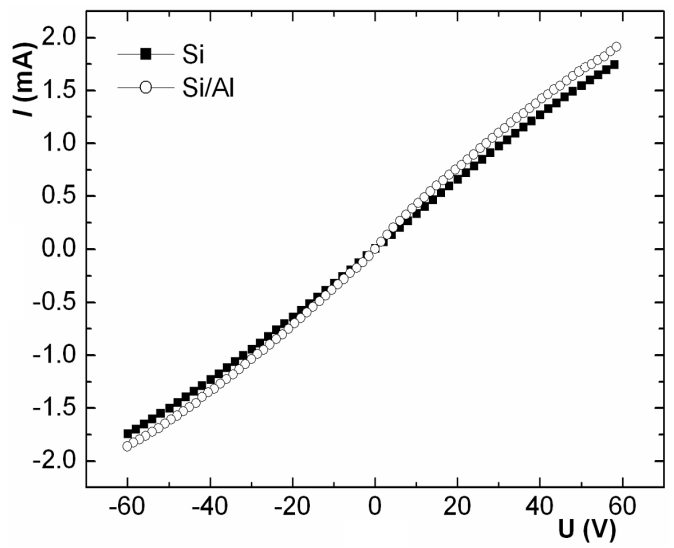

Fig. 6. $I-V$ measurement. Open circles represent sample with Al layer, closed without Al.

than "0". The discrepancy of "0" comes only from not equipotentially made Hall contacts. The $I-V$ should bring the same result, but it has to be measured more precisely. If the precision is enough, the electric current derivative $\mathrm{d} I / \mathrm{d} U$ shows bending at higher voltage $U$ as an influence of the hidden conductive layer. It has to be noted that if the diodes barrier is absent, then sample contacts become simply shorted and electric field dependent effects vanish: in that case sample parameters cannot be evaluated correctly without additional analysis. Carrier mobility can be also extracted from magnetoresistance, which in this case also shows the effect of conductive layer. From Eqs. (3.4) and (3.6) (omitting the magnetic field independent diffusion term) the current density is

$$
\boldsymbol{j}_{n}=\frac{q n \mu_{n} \boldsymbol{E}}{1+\mu_{n}^{2} B^{2}}-\frac{q n \mu_{n}^{2}[\boldsymbol{E}, \boldsymbol{B}]}{1+\mu_{n}^{2} B^{2}}+\frac{q n \mu_{n}^{3} \boldsymbol{B}(\boldsymbol{B}, \boldsymbol{E})}{1+\mu_{n}^{2} B^{2}} .
$$

The first term here gives magnetoresistance, the second - the Hall effect and the third comes from the fact that here, not as usual, electric field is redistributed by conductive layer and is not perpendicular to magnetic field in the whole crystal. The opposite magnetic field direction gives the symmetric change of magnetoresistance as it is shown in Fig. 7. Asymmetry appears because the current in magnetic field is not perpendicular to the conductive layer and is weakened by the barrier or fortified by excess conductivity at opposite sample sides, which are asymmetric as a consequence of differently shadowed sample parts with sample holders during metal evaporation. For the precise experiment, both magnetic field directions are required to measure and the mobility from magnetoresistance should be calculated after elimination of the 3rd term in (3.9) taking the average of both directions.

Electric field dependence is also available in the cases when measured material has high resistivity. The small decay of the effective Hall mobility in Fig. 1 at the voltages near to zero is probably related to the measurement specifics. When working with strong magnets (1.7 $\mathrm{T}$ in this work), switching of magnetic field pro- 


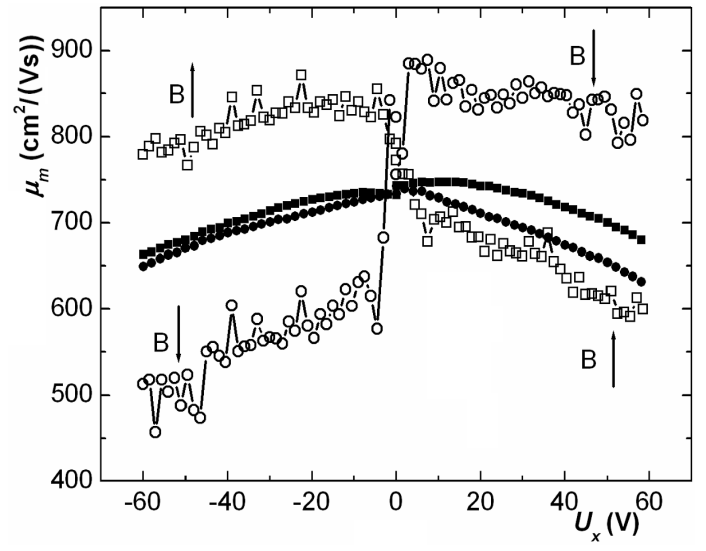

Fig. 7. Magnetoresistance effective mobility measurement. "B" indicates magnetic field induction direction. Open circles represent sample with Al layer, closed ones without $\mathrm{Al}$.

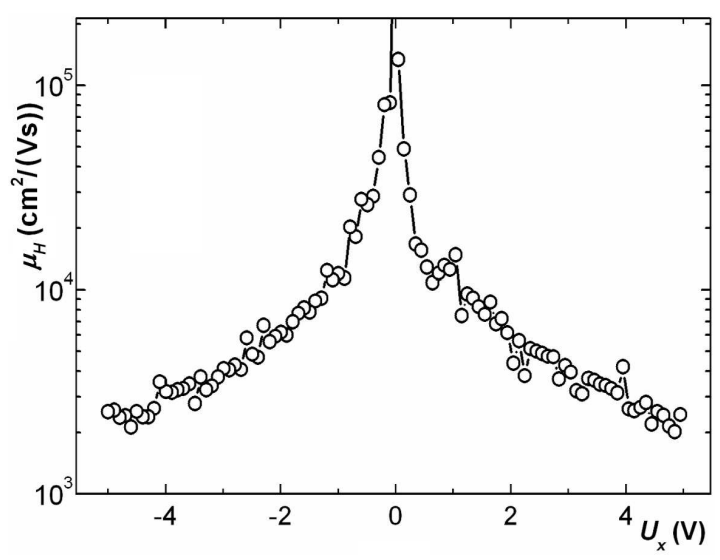

Fig. 8. Effective Hall mobility vs. applied voltage in the case of high resistive material measurement. Saturation is not achieved. The real value is found $4 \mathrm{~cm}^{2} /(\mathrm{V} \mathrm{s})$.

duces Faraday's magnetic induction, which appears in the same connecting circuit as a Hall voltage. In high resistive material the recharge is slow (through voltmeter as well) and induced voltage can remain long enough on the contacts and add a value to the Hall voltage, that way misleading Hall mobility calculation. For small applied electric fields, the Hall voltage would be small too, while the induced Faraday voltage would be electric field independent $\left(U_{\text {Faraday }} \sim \mathrm{d} B / \mathrm{d} t\right)$. The ratio $U_{\mathrm{H}} / U_{X}$ then grows as $1 / U_{X}$, when $U_{X} \rightarrow 0$ and obtained Hall mobilities are unnaturally huge like in Fig. 8 and suspected in [16]. 


\section{Summary}

The Hall mobility measurements were performed on samples with different conductivity layers and compared to homogeneous ones to find the experimental conditions when the Hall technique can be used for carrier mobility evaluation.

We recommend to perform Hall mobility dependence on applied voltage test for material characterisation whenever it is possible and analyse the obtained results according to our proposal. $I-V$ measurement test may appear as insufficient for materials (like GaN on sapphire), which have conductive layers deeper from the surface with planar contacts.

\section{References}

[1] A.J. Janavicius, J. Storasta, R. Purlys, A. Mekys, S. Balakauskas, Z. Norgela, Acta Phys. Pol. A 112, 55 (2007).

[2] C. Herring, J. Appl. Phys. 31, 1939 (1960).

[3] L.I. Shpinar, J.J. Jaskovec, Solid State Phys. 26, 1725 (1984).

[4] R.H. Bube, Appl. Phys. Lett. 13, 136 (1968).

[5] V.G. Karpov, A.Ya. Shik, B.I. Shklovsky, Sov. Phys. Semicond. 16, 1406 (1982).

[6] A. Medeišis, J. Viščakas, Lithuanian J. Phys. XV, 260 (1975).

[7] J. Viščakas, K. Lipskis, A. Sakalas, Lithuanian J. Phys. XI, 799 (1971).

[8] G.E. Stillman, S.S. Bose, M.H. Kim, B. Lee, T.S. Low, in: Handbook on Semiconductors. Characterization and Properties of Semiconductors, Eds. T.S. Moss, S. Mahajan, Elsevier, Amsterdam 1994, p. 874.

[9] A. Vandooren, S. Cristoloveanu, J.P. Colinge, in: 2000 IEEE Int. SOI Conf., Wakefield, DOI: 10.1109/SOI.2000.892798, p. 118.

[10] R.L. Petritz, Phys. Rev. 110, 1254 (1958).

[11] R.D. Larrabee, W.R. Thurber, IEEE Trans. Electron Dev. ED-27, 32 (1980).

[12] Z. Bougrioua, M. Azize, P. Gibart, T. Malinauskas, K. Neimontas, A. Mekys, J. Storasta, K. Jarašiūnas, J. Cryst. Growth 300, 228 (2007).

[13] J.H.M. ten Thije Boonkkamp, W.H.A. Shilders, in: Numerical Device Simulation, Japan Study Tour 1993, DOI: 10.1108/eb010116.

[14] V.V. Gorbachev, L.G. Spicina, Physics of Semiconductors and Metals, Metalurgia Moskva 1976, p. 369 (in Russian).

[15] A. Dargys, J. Kundrotas, Handbook on Physical Properties of Ge, Si, GaAs and InP, Science and Encyclopedia Publishers, Vilnius 1994, p. 263.

[16] H. Sitter, T. Nguyen Manh, Cryst. Res. Technol. 34, 605 (1999). 